\title{
Role of the respiratory burst in co-operative reduction in neutrophil survival by influenza $A$ virus and Escherichia coli
}

\author{
GEORG ENGELICH, MITCHELL WHITE and KEVAN L. HARTSHORN \\ Section of Hematology/Oncology, Department of Medicine and Pathology, Boston University School of \\ Medicine, 650 Albany Street, Boston, MA 02218, USA
}

\begin{abstract}
Influenza A virus (IAV)-induced impairment of neutrophil function or survival may be a cause of bacterial superinfection of IAV-infected subjects. This study was performed to determine the mechanism through which the combination of IAV and Escherichia coli co-operatively reduces neutrophil survival. Neutrophil binding of annexin-V and caspase3 activation was significantly increased by either IAV or E. coli, supporting the concept that the micro-organisms accelerate neutrophil apoptosis. The anti-apoptotic agent granulocyte-macrophage colony stimulating factor (GM-CSF) did not improve, but further reduced, survival of neutrophils treated with IAV and E. coli. As addition of $E$. coli resulted in greater neutrophil uptake of IAV and greater neutrophil respiratory burst responses to IAV, this study tested whether respiratory burst activation by IAV and $E$. coli contributes to reducing neutrophil survival. The cell-permeant NADPH oxidase inhibitor, diphenylene iodonium, significantly increased survival of neutrophils treated with either $E$. coli alone or the combination of IAV and $E$. coli. In contrast, catalase, which is not cell permeant, did not alter survival of $E$. coli- and IAV-treated neutrophils. Azide enhanced neutrophil hydrogen peroxide responses to IAV and $E$. coli, and reduced survival of these cells. These results indicate that co-operative induction of intracellular respiratory burst responses by IAV and $E$. coli mediates the reduced neutrophil survival caused by these pathogens in vitro.
\end{abstract}

\section{Introduction}

Bacterial superinfections are a major cause of morbidity and mortality during influenza A virus (IAV) epidemics, particularly among the elderly. IAV infection increases susceptibility to bacterial pneumonia, otitis media and meningitis [1]. The mechanisms through which IAV impairs host defences against bacterial infection have not been fully elucidated, but in-vitro and animal studies indicate that IAV-induced depression of neutrophil function is an important predisposing factor [2]. Recent studies also suggest that co-operative effects of IAV and bacteria on neutrophil survival may contribute to the risk of bacterial superinfection. IAV accelerates apoptosis of epithelial cells, neutrophils and monocytes in vitro [35]. Treatment of neutrophils with both IAV and

Received 26 June 2001; revised version received 17 Oct. 2001; accepted 6 Dec. 2001.

Corresponding author: Dr K. Hartshorn (e-mail: khartsho@ bu.edu).
Escherichia coli causes a rapid, marked decline in neutrophil viability [3]. This decline in viable neutrophils was much greater than that observed with either IAV or E. coli alone. Similar results were obtained with the combination of IAV and Streptococcus pneumoniae [6].

This study was undertaken to characterise the mechanisms through which IAV and E. coli co-operatively reduce neutrophil viability. First, increased annexin-V binding and caspase-3 activation were shown to occur after infection of neutrophils with IAV or E. coli. However, IAV and E. coli did not co-operatively increase caspase-3 activation. In earlier studies IAV was shown to increase neutrophil Fas antigen and Fas ligand expression; however, as in the case of caspase-3 activation, it was not possible to clearly demonstrate co-operative increases in Fas antigen or ligand expression by co-infection with IAV and E. coli [3]. Hence, this study investigated another mechanism to account for the co-operative effects of IAV and E. coli on neutrophil survival and the neutrophil respiratory burst 
response, which has been implicated as a cause of neutrophil apoptosis induced by $E$. coli, immune complexes or phorbol myristate acetate (PMA) [7-9].

\section{Materials and methods}

\section{Reagents}

Dextran, sodium citrate, RPMI-1640, trypan blue stain, Wright's-Giemsa stain, scopoletin, catalase, horseradish peroxidase type II, fMLP, diphenyleneiodonium (DPI), propidium iodide (PI) and sodium azide were purchased from Sigma. Ficoll-Paque was obtained from Pharmacia Biotech (Piscataway, NJ, USA). Dulbecco's phosphate-buffered saline (PBS) was purchased from Gibco BRL (Grand Island, NY, USA). Purified E. coli LPS was kindly provided by Dr Douglas Golenboch (Boston University School of Medicine, Boston, MA, USA).

\section{Neutrophil isolation and culture}

Neutrophils from healthy volunteer donors were isolated to $>95 \%$ purity (based on Wright's-Giemsa stain) by dextran precipitation, followed by a Ficoll-Hypaque gradient separation for removal of mononuclear cells and hypotonic lysis to eliminate contaminating erythrocytes [10]. Cell viability after isolation was $>98 \%$, as determined by trypan blue staining.

\section{Virus preparation}

Influenza A virus (Phillipines 82 H3N2 strain) was grown in the chorio-allantoic fluid of 10-day-old embryonated hen's eggs and purified on a discontinuous sucrose density gradient, as described previously [10]. Virus stocks were dialysed against PBS divided into small volumes and stored at $-80^{\circ} \mathrm{C}$ until used. Haemagglutination titres were determined by dilution of virus samples in PBS followed by addition of thoroughly washed human type $\mathrm{O}$ red blood cells. After thawing, the virus stock contained c. $5 \times 10^{8}$ plaqueforming units $/ \mathrm{ml}$ and had a protein concentration of $3.34 \mu \mathrm{g} / \mathrm{ml}$ by protein assay (BioRad).

\section{Bacterial preparations}

E. coli (K12 strain) was obtained from the American Type Culture Collection, grown in this laboratory and fixed with formalin for use in the experiments. $S$. pneumoniae suspensions in PBS were washed three times and sonicated (for three 20 -s intervals) to eliminate bacterial aggregates before each experiment. $S$. pneumoniae was pre-incubated with specific antipneumococcal $\operatorname{IgG} 32 \mu \mathrm{l} / 100 \mu \mathrm{l}$ for $30 \mathrm{~min}$ at $37^{\circ} \mathrm{C}$ and rewashed with PBS to remove residual sodium azide.
Assessment of neutrophil viability and percentage of hypodiploid neutrophils

Assessment of neutrophil viability was made after incubation of the cells for 15 min with trypan blue dye. The numbers of neutrophils that did and did not take up the dye were counted with a haemocytometer. Neutrophils that did not take up trypan blue were counted as viable. The percentage viability of neutrophils was obtained by dividing the number of viable cells by the total number of cells (i.e., both those which did and did not take up trypan blue). The percentage of cells with hypodiploid nuclei was assessed by permeabilising the cells and then exposing them to the DNAbinding dye PI. The method was performed with a slight modification of the method described by Nicoletti et al. [11]. Neutrophils were incubated with IAV or bacteria, or both, as described previously [3]. Briefly, neutrophils were incubated with either $E$. coli (or $S$. pneumoniae), IAV or a combination of IAV and the bacteria. The final approximate ratio of bacteria: neutrophil was 30:1. After incubation for $1 \mathrm{~h}$ at $37^{\circ} \mathrm{C}$ neutrophils were washed, resuspended in RPMI 1640 supplemented with autologous human serum 4\%, $20 \mathrm{mmol} / \mathrm{L}$ HEPES buffer, L-glutamine $1 \%$, penicillin $50 \mathrm{U} / \mathrm{ml}$ and streptomycin $50 \mu \mathrm{g} / \mathrm{ml}$, at $\mathrm{pH} 7.4$, and incubated at $37^{\circ} \mathrm{C}$. After incubation for $5,10,18$ and $28 \mathrm{~h}$, a fixed volume of cell suspension was removed from the culture and centrifuged at $200 \mathrm{~g}$ for $10 \mathrm{~min}$. The pellet was fixed in $1 \mathrm{ml}$ of ice-cold ethanol $70 \%$ at $4^{\circ} \mathrm{C}$ for at least $60 \mathrm{~min}$. Fixed cells were then washed with cold PBS with $\mathrm{Ca}^{2+}$ and $\mathrm{Mg}^{2+}$ and gently resuspended in $1 \mathrm{ml}$ of PI solution $(40 \mu \mathrm{g} / \mathrm{ml}$ in PBS with $\mathrm{Ca}^{2+}$ and $\mathrm{Mg}^{2+}$ ). The cells were finally incubated in the dark at room temperature for $15 \mathrm{~min}$ and analysed on a FACScan 2 flow cytometer (Becton Dickinson, San Jose, CA, USA). Forward and side scatter of neutrophils were simultaneously acquired. The red fluorescence due to PI staining of individual cells was collected under FL-2 and plotted against forward scatter. The FL-2 data were registered on a logarithmic scale. Cell debris was excluded from the analysis by appropriate raising of the forward scatter threshold. Five thousand cells of each sample were counted. All measurements were done under the same instrument settings and evaluated with the Lysis II programme.

\section{Measurement of annexin- $V$ binding}

Phosphatidylserine exposure was measured by binding of annexin-V-FITC by the protocol outlined in the annexin- $\mathrm{V}$ assay kit (Pharmingen, San Diego, CA, USA). The assay was performed as described by Fadeel et al. [9]. Briefly, this involved staining the cells with annexin and PI $(5 \mu \mathrm{g} / \mathrm{ml})$ before analysis with a FACScan flow cytometer (Becton Dickinson). Five thousand events were collected and analysed with the Cell Quest software (Becton Dickinson). Low fluorescence detritus was gated out before analysis. The 
percentage of neutrophils that had positive fluorescence and the mean neutrophil fluorescence were measured for each sample.

\section{Fluorometric analysis of caspase-3 activity}

Caspase-3 activity was measured in vitro by cleavage of the fluorogenic substrate DEVD-AMC (amino4-methylcoumarin) with a fluorometric assay kit (Pharmingen). The caspase-3 aldehyde inhibitor Ac-DEVD-CHO was used as a negative control. For preparation of cytoplasmic extracts after incubation for 5 and $18 \mathrm{~h}$, neutrophils were lysed in lysis buffer (Pharmingen). Cell lysates and substrate were combined in a reaction buffer (Pharmingen). AMC release was monitored in a spectrofluorometer at an excitation wavelength of $380 \mathrm{~nm}$ and emission wavelength of $440 \mathrm{~nm}$. Relative AMC fluorescence was expressed as the percentage of fluorescence of control cells.

\section{Assays of neutrophil $\mathrm{H}_{2} \mathrm{O}_{2}$ production and intracellular calcium release}

$\mathrm{H}_{2} \mathrm{O}_{2}$ production by neutrophils was measured by the fluorescent scopoletin assay as described previously [12]. Intracellular calcium release was measured with Fura-2 AM (Molecular Probes, Eugene, OR, USA) as described previously [10]. Briefly, neutrophils were loaded with Fura-2AM, washed and resuspended in fresh buffer. Baseline and stimulated intracellular calcium values were obtained by measuring the ratio of fluorescence at 340 and $380 \mathrm{~nm}$ with a spectrophotofluorometer.

\section{Measurement of viral uptake by neutrophils}

Neutrophils were pre-treated with bacteria or bacterial products. Viral uptake was then measured by incubating FITC-labelled IAV with neutrophils, followed by evaluation of cell-associated fluorescence by flow cytometry, as described previously [13]. Bacterial preparations were washed just before use to remove unbound FITC. Fluorescence was measured in the presence of trypan blue to quench extracellular fluorescence.

\section{Statistical methods}

Statistical significance was determined by Student's paired $t$ test. A $\mathrm{p}$ value $\leqslant 0.05$ was considered to indicate a statistically significant difference.

\section{Results}

Effects of IAV and E. coli on annexin- $V$ binding by neutrophils

As shown in Table 1, the combination of IAV and $E$. coli significantly increased the mean fluorescence of neutrophils stained with annexin-V-FITC after exposure
Table 1. Effect of IAV and E. coli on neutrophil binding of annexin-V-FITC

\begin{tabular}{lcc}
\hline $\begin{array}{l}\text { Treatment } \\
\text { group }\end{array}$ & $\begin{array}{c}\text { Mean (SEM) } \\
\text { percentage of annexin- } \\
\text { V-positive cells }\end{array}$ & $\begin{array}{c}\text { Mean (SEM) } \\
\text { annexin-V } \\
\text { fluorescence }\end{array}$ \\
\hline Control & $6.6(2.2)$ & $27.6(5)$ \\
IAV & $16(7.4)$ & $76.6(19)$ \\
E. coli & $27.8(5.8)^{*}$ & $58(20)^{*}$ \\
IAV + E. coli & $42(6.7)^{\dagger}$ & $152.5(44)^{\dagger}$ \\
\hline
\end{tabular}

Neutrophils were incubated under the indicated conditions for $5 \mathrm{~h}$, washed and labelled with annexin-V-FITC as described in Materials and methods. The percentage of neutrophils with fluorescence higher than unlabelled cells and the mean fluorescence of neutrophils were measured by flow cytometry. Results are mean and SEM of at least four experiments.

* The percentage of positive neutrophils or mean neutrophil fluorescence was significantly greater than for cells treated with control medium alone.

${ }^{\dagger}$ The percentages of annexin- $\mathrm{V}$-positive cells and mean annexin- $\mathrm{V}$ fluorescence were significantly higher in neutrophils treated with the combination of IAV and E. coli than in neutrophils treated with control medium, IAV or E. coli alone.

for $5 \mathrm{~h}$. In these samples, $42 \%$ of neutrophils were positive for annexin-V binding, compared with $16 \%$ or $28 \%$, respectively, for IAV or E. coli alone. Hence, a large proportion of these cells were undergoing early changes of apoptosis (i.e., phosphatidylserine exposure on extracellular membrane).

\section{Effects of IAV and E. coli on caspase-3 activation}

Caspase-3 activity, an indicator of apoptosis, increased in neutrophils treated with control buffer alone (i.e., from 6.4 (SEM 1.2) after incubation for $5 \mathrm{~h}$ to 15 (SEM 3.7) after $18 \mathrm{~h} ; \mathrm{n}=8 ; \mathrm{p}<0.05$ ), but treatment with either IAV or E. coli alone resulted in further significant increases (Table 2). Maximal caspase-3 activation appeared to occur after exposure for $10 \mathrm{~h}$ to IAV or E. coli, or both. Then caspase-3 activity declined so that after $18 \mathrm{~h}$ the level was no longer higher than that of buffer-treated neutrophils. Caspase3 activity in neutrophils treated with the combination of IAV and $E$. coli was not significantly greater than activity in neutrophils treated with IAV or E. coli alone (Table 2).

Table 2. Caspase-3 activity in neutrophils treated with IAV, E. coli or the combination of IAV and E. coli

\begin{tabular}{lcrc}
\hline & \multicolumn{3}{c}{$\begin{array}{c}\text { Mean (SEM) percentage of control caspase-3 activity } \\
\text { in neutrophils incubated with }\end{array}$} \\
\cline { 2 - 4 } $\begin{array}{l}\text { Incubation } \\
\text { period (h) }\end{array}$ & IAV & E. coli & IAV + E. coli \\
\hline 5 & $133(10)^{*}$ & $141(15)^{*}$ & $147(27)$ \\
10 & $181(29)^{*}$ & $247(69)^{*}$ & $233(56)^{*}$ \\
18 & $139(25)$ & $91(7)$ & $102(13)$ \\
\hline
\end{tabular}

Results shown are mean and SEM of four experiments and are expressed as the caspase- 3 activity of neutrophils treated with IAV or E. coli, or both (as indicated)/activity in neutrophils treated with control medium alone for the same period of time $\times 100$.

${ }^{*}$ Results were significantly increased as compared with neutrophils treated with control buffer alone. 
Granulocyte-macrophage colony stimulating factor (GM-CSF) and survival of neutrophils treated with the combination of IAV and E. coli

GM-CSF protects neutrophils against constitutive apoptosis, plays an important role in lung and phagocyte physiology [14] and modulates interactions of IAV with neutrophils [15]. As shown in Fig. 1a, treatment of
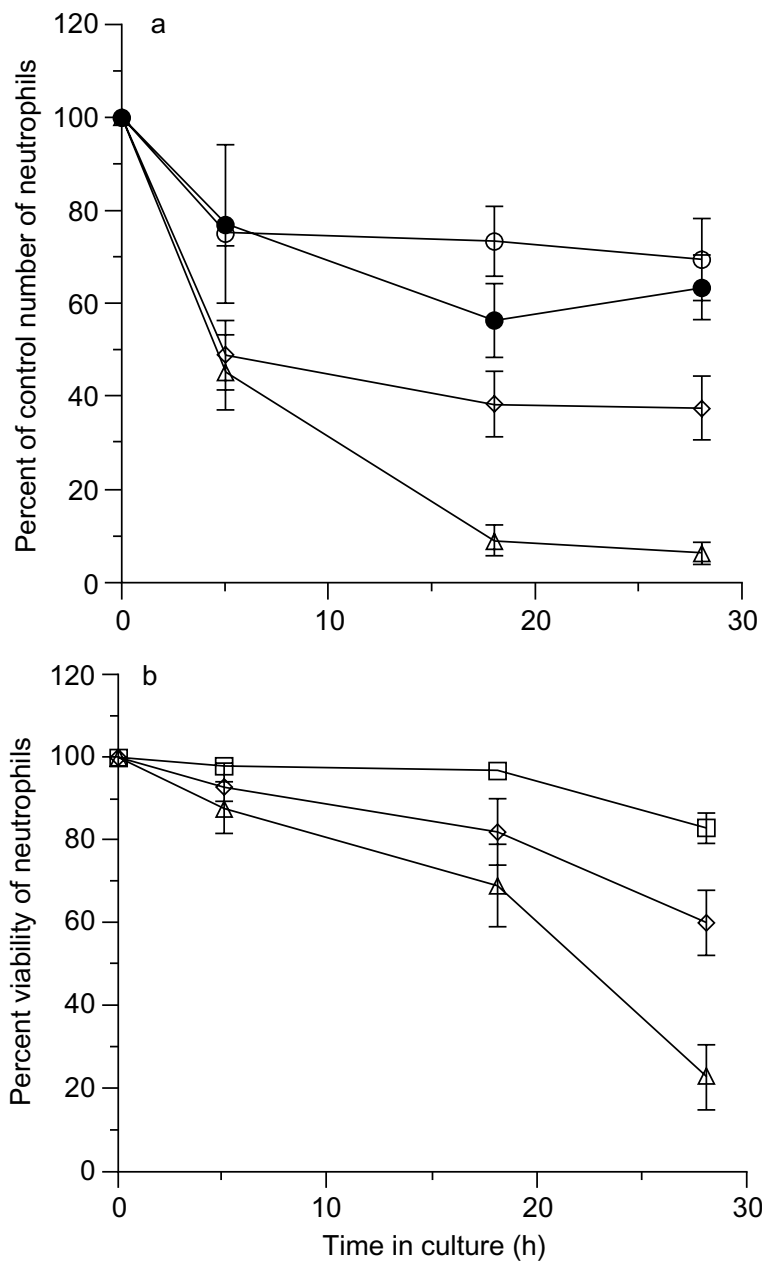

Fig. 1. Effect of GM-CSF on the survival of neutrophils in samples treated with IAV and E. coli. E. coli and IAV were incubated with neutrophils $\left(5 \times 10^{6} / \mathrm{ml}\right)$ as described in Materials and methods. (a) Mean (bar SEM) number ( $\mathrm{n} \geqslant 6$ experiments) of viable neutrophils counted in cultures treated with IAV (O) or $E$. coli $(\bullet)$ alone, the combination of IAV and $E$. coli $(\diamond)$ or the combination of IAV, E. coli and GM-CSF $(\triangle)$ after 5,18 or $28 \mathrm{~h}$ in culture. The results are expressed as percentage of control viable cells (i.e., number of viable neutrophils in cultures treated with IAV, IAV and $E$. coli, or IAV, E. coli and GM-CSF divided by viable cells in cultures maintained in control medium alone for the same duration $\times 100$ ). For cultures treated with the combination of IAV and E. coli, viable cell counts were significantly less $(\mathrm{p}<0.05)$ than for those treated with control medium, IAV or E. coli alone at all time points. (b) Percentage of viable neutrophils defined as percentage of cells able to exclude trypan blue in cultures treated with control medium alone ( $\square)$, IAV and E. coli $(\diamond)$, or IAV, E. coli and GM-CSF $(\triangle)$. The results were obtained by dividing the number of cells that excluded trypan blue by the total number of cells in each culture. Addition of GM-CSF reduced the percentage of viable neutrophils compared with treatment with IAV and E. coli alone $(\mathrm{p}<0.05)$. neutrophils with IAV or E. coli, or both, caused a marked decline in the number of surviving neutrophils after 18 or $28 \mathrm{~h}$, as compared with neutrophils treated with control buffer alone. As previously reported, the combination of IAV and $E$. coli caused a significantly greater reduction in surviving neutrophils than either IAV or E. coli alone. Addition of GM-CSF did not improve survival of neutrophils treated with the combination of IAV and E. coli. In fact, after $28 \mathrm{~h}$ there were significantly fewer surviving cells in samples treated with IAV, E. coli and GM-CSF as compared with those treated with IAV and E. coli. The percentage of viable neutrophils also showed that GMCSF worsened survival of neutrophils treated with IAV and E. coli (Fig. 1b).

\section{Effects of E. coli on neutrophil uptake of IAV}

An earlier study reported that treatment of neutrophils with IAV increases subsequent uptake of $E$. coli [3]. Treatment of neutrophils with $E$. coli also increased uptake of FITC-labelled IAV by the cells (Table 3). In contrast, $S$. pneumoniae did not significantly increase uptake of IAV by neutrophils. The effect of E. coli could not be attributed to E. coli-derived LPS alone, as purified LPS did not increase IAV uptake (Table 3). A previous study demonstrated that GM-CSF increases neutrophil uptake of IAV [15]. However, GM-CSF did not cause a significantly greater increase in IAV uptake when added along with IAV and E. coli (Table 3).

Evidence for a role of respiratory burst activation in co-operative induction of apoptosis by IAV and E. coli

Previous studies have also demonstrated that the combination of IAV and E. coli triggers a greater respiratory burst response than either agent alone [3]. Hence, agents that inhibit or enhance the respiratory burst were tested for their ability to affect the survival of neutrophils treated with IAV or E. coli, or both. Pretreatment with DPI, a cell-permeant inhibitor of NADPH oxidase activation, fully inhibited $\mathrm{H}_{2} \mathrm{O}_{2}$ responses and reduced intracellular calcium responses

Table 3. Effect of E. coli, S. pneumoniae, LPS and GMCSF on neutrophil uptake of FITC-labelled IAV

\begin{tabular}{lc}
\hline Stimulus & $\begin{array}{c}\text { Mean (SEM) } \\
\text { IAV binding (\%) }\end{array}$ \\
\hline Control & 100 \\
E. coli & $209(8)^{*}$ \\
E. coli + GM-CSF & $251(37)^{*}$ \\
LPS & $101(7.5)$ \\
S. pneumoniae & $116(24)$ \\
S. pneumoniae + GM-CSF & $124(13)$ \\
\hline
\end{tabular}

Results represent mean \pm SEM $(n=3)$ of neutrophil fluorescence after treatment with FITC-labelled IAV and are expressed as percentage of IAV binding (as compared with binding of FITClabelled IAV to untreated neutrophils).

${ }^{*} \mathrm{p}<0.05$ compared with binding of FITC-labelled IAV alone. 
of neutrophils treated with IAV and $E$. coli (Table 4). DPI significantly reduced the development of hypodiploid neutrophils in cultures treated with $E$. coli alone or the combination of IAV and E. coli (Fig. 2) such that these cultures were identical to controls. DPI did not

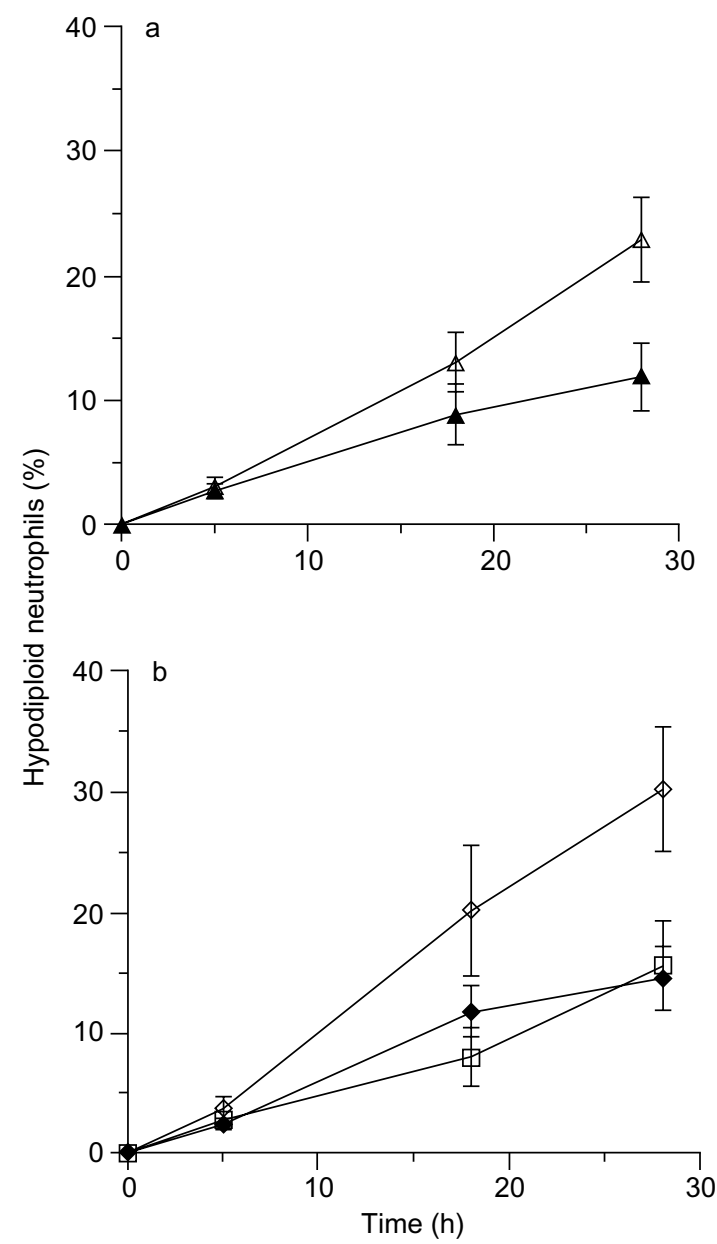

Fig. 2. The effect of the NADPH oxidase inhibitor DPI on viability of neutrophils treated with $E$. coli alone (a) or the combination of IAV and $E$. coli $(\mathbf{b})$. Neutrophils $\left(5 \times 10^{6} / \mathrm{ml}\right)$ were incubated with IAV or $E$ coli, or both, in the absence or presence of DPI $(10 \mu \mathrm{mol} / \mathrm{L})$. DPI was added $10 \mathrm{~min}$ before IAV or E. coli. The percentage of cells displaying hypodyploid DNA was measured by flow cytometry. $\triangle, E$. coli alone; $\boldsymbol{\Delta}, E$. coli + DPI; $\square$, control medium; $\diamond, E$. coli + IAV + DPI; $\diamond, E$. coli + IAV. Results represent mean (SEM) of four separate experiments. $\left(^{*}\right)$ indicates instances in which DPI significantly improved the viability of neutrophils $(\mathrm{p}<0.05)$. significantly alter the viability of control neutrophils or neutrophils treated with IAV alone (data not shown).

Although catalase blocks detectable $\mathrm{H}_{2} \mathrm{O}_{2}$ in the extracellular media of neutrophils stimulated with IAV, it does not inhibit chemiluminescent responses to the virus [16]. Thus, the IAV-induced respiratory burst response largely originates at an intracellular location and is not inhibited by catalase because catalase is not cell permeant. Catalase also did not inhibit the generation of hypodiploid neutrophils in cultures treated with IAV and E. coli. The mean percentages of hypodiploid neutrophils after 18 and $28 \mathrm{~h}$ were 32 (SEM 14) and 55 (SEM 18), respectively, in the absence of catalase and 30 (SEM 11) and 63 (SEM 15), respectively, in the presence of catalase $(\mathrm{n}=4)$.

Azide, a cell-permeant inhibitor of catalase and myeloperoxidase which enhances detectable $\mathrm{H}_{2} \mathrm{O}_{2}$ release from IAV-treated neutrophils [16], also increased $\mathrm{H}_{2} \mathrm{O}_{2}$ release after stimulation with $E$. coli or the combination of IAV and E. coli. The response to E. coli or the combination of IAV and E. coli was increased by 67 (SEM 14)\% and 50 (SEM 7)\%, respectively $(\mathrm{n}=4$; $\mathrm{p}<0.005$ for each). Azide also significantly increased the percentage of hypodiploid cells in samples treated with IAV and E. coli (Fig. 3) but not in samples maintained in control buffer alone (Fig. 3).

\section{Discussion}

In previous studies, incubation with IAV or E. coli alone caused an acceleration of neutrophil apoptosis [3,7], and this was increased on incubation with both IAV and E. coli. Also, initial studies at this laboratory demonstrated that either IAV or E. coli increased Fas antigen expression on neutrophils and IAV caused significantly increased Fas ligand expression on neutrophils [3]. However, the combination of IAV and E. coli did not clearly increase Fas antigen or Fas ligand expression to a greater extent than either pathogen alone.

In searching for the mechanisms of the co-operative adverse effect of IAV and E. coli on neutrophil

Table 4. Effect of DPI on neutrophil $\mathrm{H}_{2} \mathrm{O}_{2}$ production or intracellular calcium rise in response to IAV or the combination of IAV and E. coli

\begin{tabular}{|c|c|c|c|c|}
\hline \multirow[b]{2}{*}{ Stimulus } & \multicolumn{2}{|c|}{ Mean (SEM) $\mathrm{H}_{2} \mathrm{O}_{2}$ production } & \multicolumn{2}{|c|}{ Mean (SEM) calcium response } \\
\hline & DPI- & DPI+ & DPI- & DPI+ \\
\hline IAV & $1.0(0.3)$ & 0 & $76.5(9)$ & $60(7)$ \\
\hline $\mathrm{IAV}+E . c o l i$ & $2(0.4)$ & $0.04(0.04)$ & 91 (17) & $46(12)$ \\
\hline fMLP & $2(0.4)$ & $0.2(0.04)$ & $\ldots$ & $\ldots$ \\
\hline
\end{tabular}

After incubation with or without DPI $(10 \mu \mathrm{M} / \mathrm{L})$ neutrophils were stimulated with IAV (Phil82H3N2) or E. coli (K12), or both, or fMLP $\left(10^{-7} \mathrm{M}\right)$. Results are mean (SEM) maximal rate of $\mathrm{H}_{2} \mathrm{O}_{2}$ production in $\mathrm{nmol} / \mathrm{min} / 4 \times 10^{6}$ cells $(\mathrm{n}=3)$ and maximal rise in intracellular calcium as measured by Fura-2AM $(n=3)$. DPI significantly reduced $\mathrm{H}_{2} \mathrm{O}_{2}$ and calcium responses to all stimuli tested $(\mathrm{p}<0.05)$. 


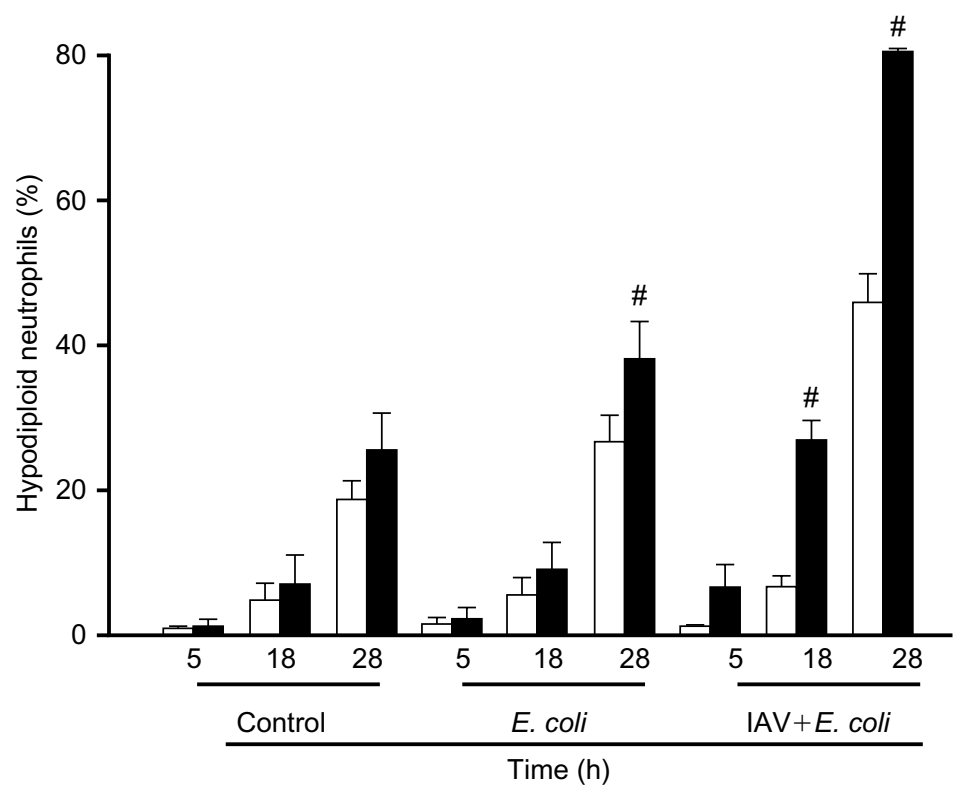

Fig. 3. Effect of sodium azide, a blocker of intracellular catalase and peroxidases, on IAV- and $E$. coli-induced generation of hypodiploid neutrophils. Neutrophils $\left(5 \times 10^{6} / \mathrm{ml}\right)$ were incubated with IAV and E. coli in the absence $(\square)$ or presence $(\mathbf{\square})$ of sodium azide $(1 \mathrm{mM})$ for indicated times. The percentage of cells displaying hypodiploid DNA was measured by flow cytometry. Results represent mean (SEM) of three separate experiments. (\#) indicates where addition of sodium azide significantly increased generation of hypodiploid neutrophils ( $p<0.05$ ).

survival, this study demonstrated that the combination of these micro-organisms increased annexin- $\mathrm{V}$ expression significantly more than either IAV or $E$. coli alone after incubation for $5 \mathrm{~h}$. These results indicate that IAV and $E$. coli modify neutrophil membrane phosphatidylserine expression relatively rapidly in vitro and support the concept that these micro-organisms cooperatively induce neutrophil apoptosis. IAV or E. coli also increased caspase-3 activation in neutrophils after $5 \mathrm{~h}$. Therefore, increased annexin- $\mathrm{V}$ binding and caspase-3 activation preceded the marked decline in neutrophil viability observed in cultures treated with IAV and E. coli (Fig. 1b). However, the caspase-3 assay (like the Fas ligand and Fas antigen assays noted above) did not show co-operative effects of IAV and $E$. coli. Hence, mechanisms other than Fas signalling or caspase- 3 activation appear to be involved in the cooperative induction of apoptosis by IAV and E. coli.

The study next attempted to modulate survival of neutrophils treated with IAV and E. coli with the antiapoptotic agent GM-CSF, but it caused a further reduction in survival of IAV- and E. coli- treated neutrophils with greater evidence of cell necrosis. Although the mechanism of this effect of GM-CSF is unclear it may relate to its ability to increase neutrophil respiratory burst responses to IAV [15] (see below).

The present study evaluated whether the effects of $E$. coli on neutrophil uptake of IAV may contribute to cooperative reduction in neutrophil survival caused by IAV and E. coli. A previous study found that IAV increases neutrophil uptake of and respiratory burst responses to E. coli [3] and, as shown in Table 3, there is also a reciprocal effect through which E. coli increases neutrophil uptake of IAV. Also, it has been shown that the respiratory burst response triggered by IAV occurs mostly at an intracellular location [16] and recent studies have indicated that neutrophil respiratory burst responses (especially those occurring intracellularly [17]) are an important trigger for neutrophil apoptosis. Therefore, it was speculated that the ability of IAV and E. coli to reciprocally increase their uptake or co-operative enhancement of respiratory burst responses could account for co-operative increases in apoptosis.

The use of several biochemical modulators provided strong evidence for a critical role of intracellular respiratory burst products in mediating the reduction in neutrophil survival caused by the combination of IAV and E. coli. The cell-permeant respiratory burst inhibitor DPI significantly improved the viability of neutrophils treated with $E$. coli or the combination of IAV and E. coli, without affecting the viability of neutrophils maintained in control buffer. Furthermore, azide, an inhibitor of catalase and peroxidases which is also cell permeant and potentiates $\mathrm{H}_{2} \mathrm{O}_{2}$ production in response to these stimuli, markedly potentiated IAVand E. coli-mediated reduction in neutrophil survival (again without altering survival of control neutrophils). Finally catalase, which catalyses the breakdown of $\mathrm{H}_{2} \mathrm{O}_{2}$ but which is not able to permeate the cell membrane, did not alter the effects of IAV and E. coli on neutrophils. These results indicate that the ability of E. coli alone or in combination with IAV to reduce neutrophil survival in vitro involves generation of respiratory burst products within the neutrophil. 
Furthermore, as azide inhibits myeloperoxidase, the effect does not involve products formed by myeloperoxidase (e.g., $\mathrm{HOCl}^{-}$).

It remains to be determined whether the ability of IAV and bacteria to co-operatively reduce neutrophil survival in vitro is relevant to the clinical observation that IAV predisposes to bacterial superinfections. Although $E$. coli is not an organism that is commonly isolated from IAV-infected patients who develop bacterial pneumonia, the more relevant $S$. pneumoniae also markedly reduced neutrophil survival when added along with IAV in vitro through a respiratory burstrelated mechanism [6]. It has been demonstrated in murine models that measures which reduce the production of oxidants reduce the severity and mortality of influenza pneumonia [18]. Investigations should be done to determine whether similar findings would be obtained in models of co-infection with IAV and bacteria.

This work was supported by NIH grant HL 58910 to K.L.H.

\section{References}

1. Hartshorn KL. Etiology of bacterial superinfections complicating influenza viral infection. In: Brown LE, Hampson AW, Webster RG (eds) Options for the control of influenza III. Amsterdam, Elsevier Science. 1996: 499-508.

2. Abramson JS, Mills EL. Depression of neutrophil function induced by viruses and its role in secondary microbial infections. Rev Infect Dis 1988; 10: 326-341.

3. Colamussi ML, White MR, Crouch E, Hartshorn KL. Influenza A virus accelerates neutrophil apoptosis and markedly potentiates apoptotic effects of bacteria. Blood 1999; 93: $2395-2403$.

4. Mori I, Komatsu T, Takeuchi K, Nakakuki K, Sudo M, Kimura Y. In vivo induction of apoptosis by influenza virus. $J$ Gen Virol 1995; 76: 2869-2873.
5. Morris SJ, Price GE, Barnett JM, Hiscox SA, Smith H, Sweet C. Role of neuraminidase in influenza virus-induced apoptosis $J$ Gen Virol 1999; 80: 137-146.

6. Engelich G, White M, Hartshorn KL. Neutrophil survival is markedly reduced by incubation with influenza virus and Streptococcus pneumoniae: role of respiratory burst. J Leukoc Biol 2001; 69: $50-56$

7. Watson RWG, Redmond HP, Wang JH, Condron C, BouchierHayes D. Neutrophils undergo apoptosis following ingestion of Escherichia coli. J Immunol 1996; 156: 3986-3992.

8. Gamberale R, Giordano M, Trevani AS, Andogenui G, Geffner JR. Modulation of human neutrophil apoptosis by immune complexes. J Immunol 1998; 161: 3666-3674.

9. Fadeel B, Åhlin A, Henter J-I, Orrenius S, Hampton MB. Involvement of caspases in neutrophil apoptosis: regulation by reactive oxygen species. Blood 1998; 92: 4808-4818.

10. Hartshorn KL, Collamer M, Auerbach M, Myers JB, Pavlotsky $\mathrm{N}$, Tauber AI. Effects of influenza A virus on human neutrophil calcium metabolism. J Immunol 1988; 141: 1295 1301.

11. Nicoletti I, Migliorati G, Pagliacci MC, Grignani F, Riccardi C. A rapid and simple method for measuring thymocyte apoptosis by propidium iodide staining and flow cytometry J Immunol Methods 1991; 139: 271-279.

12. Hartshorn KL, Collamer M, White MR, Schwartz JH, Tauber AI. Characterization of influenza A virus activation of the human neutrophil. Blood 1990; 75: 218-226.

13. Hartshorn KL, Crouch E, White MR et al. Pulmonary surfactant proteins $\mathrm{A}$ and $\mathrm{D}$ enhance neutrophil uptake of bacteria. Am J Physiol 1998; 274: L958-L969.

14. Dranoff $\mathrm{G}$, Crawford $\mathrm{AD}$, Sadelain $\mathrm{M}$ et al. Involvement of granulocyte-macrophage colony-stimulating factor in pulmonary homeostasis. Science 1994; 264: 713-716.

15. Little R, White MR, Hartshorn KL. Interferon-alpha enhances neutrophil respiratory burst responses to stimulation with influenza A virus and FMLP. $J$ Infect Dis 1994; 170: 802-810.

16. Kazhdan M, White MR, Tauber AI, Hartshorn KL. Human neutrophil respiratory burst response to influenza A virus occurs at an intracellular location. J Leukoc Biol 1994; 56: $59-64$.

17. Lundqvist-Gustafsson $\mathrm{H}$, Bengtsson $\mathrm{T}$. Activation of the granule pool of the NADPH oxidase accelerates apoptosis in human neutrophils. J Leukoc Biol 1999; 65: 196-204.

18. Akaike T, Noguchi Y, Ijiri S et al. Pathogenesis of influenza virus-induced pneumonia: involvement of both nitric oxide and oxygen radicals. Proc Natl Acad Sci USA 1996; 93: 24482453. 